\title{
Caracterización física, nutricional y no nutricional de las semillas de Inga paterno
}

\author{
Physical, nutritional and non nutritional \\ characterization of Inga paterno seeds
}

\begin{abstract}
Inga paterno is a tree legume present in various states of Mexico whose sheath is consumed in some places as a fruit due seed coat is sweet (Aryl). Seeds are not commonly consumed and there are few studies about its composition. The physical characteristics, nutritional and non-nutritional composition of Inga paterno seeds from México State and Puebla were analyzed. The seeds showed a significant proportion of protein and lipid. The oil from the seeds showed higher proportion of palmitic acid. Non-nutritional compounds such as phenolics, tannins, phytates, saponins and trypsin inhibitors were quantified. The seeds of the two sources of origin showed no statistically significant difference in their physical, chemical and non-nutritional characteristics; however, unlike other legumes, they exhibit high saponins content and high inhibitory trypsin activity.

Key words: Legumes, Inga paterno, nutritional composition, non nutritional compound.
\end{abstract}

Noemi A Sánchez Mendoza (1)

Cristian Jiménez Martínez (1)

Anaberta Cardador Martínez (2)

Sandra Martín del Campo Barba (2)

Gloria Dávila Ortiz 81)

Este trabajo fue recibido el 14 de Abril de 2016 y aceptado para ser publicado el 28 de Septiembrede 2016.

\section{INTRODUCCIÓN}

Uno de los problemas más importantes a los que se enfrenta la humanidad es el desabastecimiento de alimentos por la insuficiente producción y distribución, pese a los avances tecnológicos actuales [1]. Una de las opciones que han surgido con un importante impacto y buena aceptación, es el uso de alternativas alimenticias no convencionales. El grupo de especies al que se ha dedicado gran parte de la investigación en este aspecto, es el de las leguminosas, pues proporcionan energía debido a su importante contenido de carbohidratos (50 a 60\%) además de la capacidad de sintetizar y almacenar proteínas (20 a 40\%), convirtiéndose en fuente importante de éstos. Como ejemplos podemos encontrar al frijol (Phaseolus vulgaris), el garbanzo (Cicer arietinum), el haba (Vicia faba) y el chícharo (Pisum sativum) [2, 3].

Uno de los tópicos actuales es empleary dar un uso adicional a las leguminosas de consumo no convencional, buscando satisfacer una demanda alimentaria que crece a grandes pasos. Las leguminosas son de gran importancia nutricional debido a su disponibilidad y a que contiene además de carbohidratos, una buena cantidad de proteína y vitaminas del complejo
B. Algunas como el cacahuate (Arachis hypogaea) y la soya (Glycine max) son además ricas en aceite. En general, todas las leguminosas son un buen suplemento en las dietas basadas en cereales, ya que cuando las semillas comestibles y los cereales se consumen en una misma comida, suministran una mezcla de proteínas con una mayor variedad de aminoácidos, lo que mejora el valor proteico de la dieta [4]. Sin embargo, el uso de estas leguminosas se ve limitado por la presencia de compuestos no nutricionales, llamados así porque interfieren o dificultan la asimilación por los organismos vivos de algunos nutrientes y en algunos casos pueden llegar a ser tóxicos o causar efectos fisiológicos poco deseables [5, 6], siendo importante la concentración en la que se encuentran, para de ser necesario, aplicar métodos que permitan disminuirlos o eliminarlos. Sin embargo, estos compuestos en pequeñas cantidades, pueden ser también beneficiosos para la salud en la prevención de diversas enfermedades, por lo que actualmente se les ha denominado compuestos bioactivos, fitoquímicos o no nutricionales ya que, si bien carecen de valor nutritivo, no siempre resultan perjudiciales. Desde el punto de vista bioquímico los compuestos no nutricionales son de naturaleza 
variada, no aparecen por igual en todas las plantas y sus efectos fisiológicos son también diferentes, por lo que las leguminosas son consideradas como alimentos funcionales $[7,8]$.

Inga es un grupo de especies que pertenece a la familia de las leguminosas, y en específico a la de las fabaceaemimosideae. Son árboles de tamaño mediano a grande $y$ pueden diferenciarse por el tamaño y forma de la hoja, por la copa y la tolerancia al tipo de suelo. Su presencia en el continente americano esta reportada desde México hasta Argentina, además de las Antillas [9]. La reproducción es por semillas, las cuales son sensibles al calor [10]. Producen frutos en forma de vainas que se abren longitudinalmente a lo largo de dos suturas. Su cultivo no está ampliamente distribuido ni se hace de manera comercial; sin embargo, contribuyen al sustento de las comunidades que colectan sus vainas y las comercializan como fruto, consumiéndose principalmente la pulpa algodonosa, también llamada arilo y en menor medida las semillas [11], pues una vez consumida la pulpa, la vaina y la semilla son generalmente desechadas.

En la actualidad, son realmente escasos los estudios acerca de las propiedades nutricionales y funcionales que podría presentar la semilla de Inga, por lo que en este trabajo se analizó su contenido de compuestos nutricionales y no nutricionales para poder contemplarla como una opción alimenticia y/o funcional.

\section{MATERIALES Y MÉTODOS Material biológico}

Las semillas de Inga paterno se obtuvieron a partir de frutos colectados del municipio de Ozumba de Alzate (19 $02^{\prime} 21^{\prime \prime} \mathrm{N}$ y $98^{\circ} 47^{\prime} 37^{\prime \prime} \mathrm{O}$, altitud 2,340 msnm) en el Estado de México, durante la temporada de otoño (mes de octubre) de 2012 y en el municipio de Tochimilco (18 $53^{\prime} 38^{\prime \prime} \mathrm{N}$ y 98 34'22'O, altitud 2081 msnm) en el Estado de Puebla, durante el Verano (mes de julio) de 2013. Durante estos meses pueden encontrarse las vainas en los mercados locales.

Las vainas fueron abiertas para retirar manualmente las semillas y la capa de pulpa (arilo). Después fueron lavadas con agua destilada para retirar residuos de pulpa, se secaron a temperatura ambiente durante 96 h o hasta alcanzar una humedad menor al 10\%. Posteriormente fueron trituradas en un molino, hasta obtener harina con un tamaño de partícula homogéneo (tamiz 45-350).

Caracterización física de las semillas y las vainas

Las semillas de Inga enteras y sin daños, frescas y secas, además de las vainas fueron colocadas en una lámina plástica transparente y se obtuvo una imagen mediante un escáner (Hewlett Packard 5280, resolución 4800 x 4800 dpi óptica). La imagen obtenida se procesó con el software ImageJ $1.45 \mathrm{~s}$ (Wayne Rasband National Institutes of Health, USA) para obtener los resultados de eje mayor y menor.

\section{Composición química de las harinas}

La caracterización química de la harina de las semillas secas se realizó de acuerdo a los siguientes métodos: proteína por Kjeldhal (NX6.25), lípidos (920.39) fibra cruda total (958.29) y cenizas (923.03) (A.O.A.C, 2005); Carbohidratos por el método de la Antrona [12]. La determinación de humedad se hizo empleando una termobalanza (Ohaus MB45).

Identificación de ácidos grasos

Las muestras del extracto etéreo de las semillas obtenido mediante extracción sólido-líquido en soxhlet se analizaron según el método descrito por Martín del Campo [13] utilizando un sistema de cromatografía de gases (GC) capilar Agilent 7890A (Agilent Technologies, Palo Alto, CA) acoplado a espectrometría de masas (Agilent 5975C). Los compuestos se separaron en una columna HP-88 ((88\% - Cyanopropy) aryl-polysiloxane, $0.25 \mathrm{~mm} \times 30-100 \mathrm{~m} \times 0.20 \mu \mathrm{m}$ Agilent Technologies) que permitió la separación de los ésteres metílicos de ácidos grasos (FAMEs). La identificación se llevó a cabo con la biblioteca NIST 05. La cuantificación se realizó de acuerdo al porcentaje de área.

\section{CONTENIDO DE COMPUESTOS NO NUTRICIONALES DE LAS HARINAS}

Determinación del contenido de fenólicos totales

El contenido de fenólicos totales fue determinado a las harinas mediante el método de Folin-Cioucalteu [14] usando ácido gálico como estándar. La absorbancia fue medida a 760 $\mathrm{nm}$ contra agua destilada como blanco. El contenido de fenólicos totales fue expresado como equivalentes de ácido gálico mediante una curva de calibración. El intervalo de linealidad de la curva de calibración fue de 0 a $0.5 \mathrm{mg} / \mathrm{mL}(r=0.996)$.

Determinación de taninos condensados

El contenido de Taninos se determinó mediante el método de la vainillina [15] usando (+)-catequina como estándar. La absorbancia se midió a $500 \mathrm{~nm}$ contra un blanco de reactivos ( $0.5 \mathrm{~mL}$ de metanol al $80 \%+2.5 \mathrm{~mL}$ de vainillina al $1 \%+2.5$ $\mathrm{mL}$ de $\mathrm{HCl}$ al 8\%). El contenido de taninos se expresó como equivalentes de (+)-catequina mediante una curva de calibración. El intervalo de linealidad de la curva de calibración fue de 0 a $1.0 \mathrm{mg} / \mathrm{mL}(\mathrm{r}=0.998)$.

\section{Determinación de fitatos}

Se determinó el contenido de fitatos mediante el método de Vaintraub y Latpeva [16] empleando como estándar ácido fítico. La absorbancia se midió a $500 \mathrm{~nm}$ contra agua destilada como blanco. El contenido de fitatos se expresó como equivalentes de ácido fítico mediante una curva de calibración. El intervalo de linealidad de la curva de calibración fue de 0 a $0.160 \mathrm{mg} / \mathrm{ml}(\mathrm{r}=0.997)$.

\section{Determinación de inhibidores de tripsina}

La cuantificación de la actividad de inhibición de tripsina se realizó de acuerdo a los ensayos de Smith et al., [17] modificado, agregando la enzima en el último momento de acuerdo a Liu and Markakis [18]. Se reporta la actividad de inhibidores de tripsina en términos de $\mathrm{mg}$ de tripsina pura inhibida /g muestra.

\section{Determinación de saponinas totales}

La cuantificación de las saponinas presentes en la muestra se realizó de acuerdo al método de Hiai et al., [19] empleando diosgenina como estándar. La absorbancia fue medida a 520 $\mathrm{nm}$ contra agua destilada como blanco. Se reportó el contenido de saponinas como equivalentes de diosgenina mediante una curva de calibración, utilizando concentraciones de 0 a 0.125 $\mathrm{mg}(\mathrm{r}=0.997)$.

Identificación y cuantificación de $\alpha$-galactósidos

La identificación y cuantificación de los a-galactósidos se hizo de acuerdo a los métodos de Muzquiz et al., [20] y de Johansen et al. [21] con algunas modificaciones. La cuantificación se realizó por HPLC, utilizando un equipo Agilent Tecnologies Serie 1200 (Alemania) equipado con una 
columna Zorbax (Carbohydrate Analysis Column), la fase móvil fue acetonitrilo:agua (60:40, v/v), en condiciones isocráticas con un flujo de $1.0 \mathrm{~mL} / \mathrm{min}$ durante $15 \mathrm{~min}$ con un volumen de inyección de $20 \mu \mathrm{L}$. Se utilizó un detector de índice de refracción Agilent Serie 1200 (para el rango de índices de refracción:1,00-1,75 calibrado). Se emplearon patrones externos de rafinosa, estaquiosa y verbascosa.

\section{ANÁLISIS ESTADÍSTICO}

Cada ensayo de cuantificación de los componentes nutricionales y no nutricionales se realizó por triplicado. Se realizó un análisis de varianza unifactorial (ANOVA). El nivel de significancia de las diferencias se definió como $p<0.05$. El análisis estadístico se realizó con el software Stat Graphics Centurion XV.

\section{RESULTADOS Y DISCUSIÓN}

Análisis de imagen

Las vainas y las semillas fueron escaneadas y procesadas con el software Image J 1.45s, obteniéndose las imágenes que se muestran en la figura 1.

El eje mayor de las vainas provenientes de Ozumba (Cuadro 1) fue de 203 mm, mientras que el eje menor fue de 33.9 $\mathrm{mm}$. El eje mayor de las semillas frescas procedentes de esta región fue de $22.5 \mathrm{~mm}$ y el eje menor de $13.6 \mathrm{~mm}$, es decir, una relación largo/ancho de 1.65. Una vez que se secaron las semillas, el eje mayor fue de $16.1 \mathrm{~mm}$ mientras que el eje menor fue de $9.2 \mathrm{~mm}$, siendo la relación largo/ancho de 1.74 .

Por otra parte, el eje mayor de las vainas provenientes de Tochimilco (Cuadro 1) fue de $232 \mathrm{~mm}$ y de $35.4 \mathrm{~mm}$ para el eje menor, respectivamente. El eje mayor de las semillas frescas fue de $24.8 \mathrm{~mm}$ y el menor fue de $15.2 \mathrm{~mm}$, es decir, una relación largo/ancho de 1.63. El eje mayor de las semillas secas fue de $19.1 \mathrm{~mm}$ mientras que el eje menor fue de 10.89 $\mathrm{mm}$, siendo la relación largo/ancho de 1.75 .

Como se puede observar (cuadro 1), no existió una diferencia estadísticamente significativa entre las dimensiones de las vainas de ambos lugares; sin embargo, las semillas, si mostraron diferencia estadísticamente significativa, ya que las semillas de Tochimilco son más largas y anchas que las

\section{FIGURA 1}

Vainas frescas, semillas frescas y semillas secas de Inga paterno de (a) Ozumba de Alzate, Estado De México y (b) Tochimilco, Puebla.

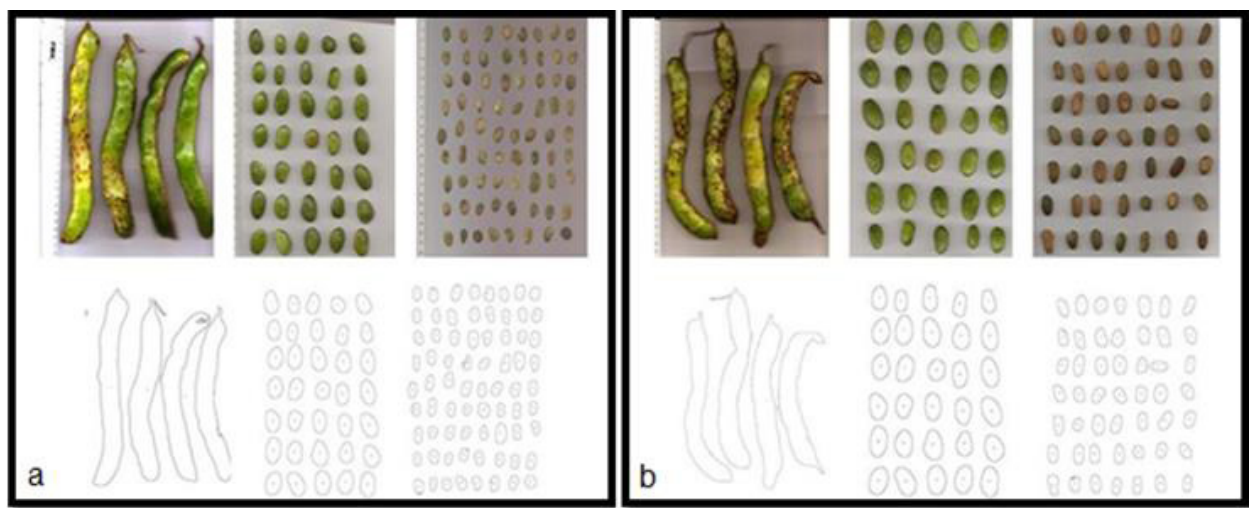

\section{CUADRO 1}

Características físicas de las vainas, semillas frescas y secas de Inga paterno.

\begin{tabular}{|c|c|c|c|}
\hline & & Ozumba & Tochimilco \\
\hline \multirow[t]{2}{*}{ Vaina } & Eje mayor & $203 \pm 3.7^{a}$ & $232 \pm 1.9^{a}$ \\
\hline & Eje menor & $33.9 \pm 1.7^{a}$ & $35.4 \pm 0.7^{a}$ \\
\hline \multirow[t]{2}{*}{ Semillas frescas } & Eje mayor & $22.5 \pm 2.5^{a}$ & $24.8 \pm 2.4^{b}$ \\
\hline & Eje menor & $13.6 \pm 1.5^{a}$ & $15.2 \pm 1.0^{b}$ \\
\hline \multirow[t]{2}{*}{ Semillas secas } & Eje mayor & $16.1 \pm 1.8^{a}$ & $19.1 \pm 2.1^{b}$ \\
\hline & Eje menor & $9.2 \pm 1.1 \mathrm{a}$ & $10.9 \pm 1.1^{b}$ \\
\hline
\end{tabular}

Resultados expresados en $\mathrm{mm}$. Letras diferentes en la misma fila indican diferencia significativa $(p<0.05)$ 
de Ozumba, tanto frescas como secas. Niembro [22] reportó dimensiones de 24 a $32 \mathrm{~mm}$ de largo y de 12 a $18 \mathrm{~mm}$ de ancho para semillas frescas de Inga jinicuil, resultados similares a los reportados en este trabajo.

Existen diversas opiniones respecto a la influencia del tamaño como factor de calidad en las semillas ya que mientras algunos autores afirman que conocer el tamaño de la semilla es importante debido a que se ha relacionado con la calidad fisiológica de la misma para distintas especies [23]; sin embargo, existen otros estudios que afirman que la calidad fisiológica no depende del tamaño de la semilla [24].

\section{Composición química}

Se determinó la humedad de las muestras de Inga paterno para asegurar que el contenido fuera inferior o igual al 10\%, ya que a humedades mayores se facilita la proliferación de microorganismos y la actividad enzimática deteriorativa [25].

En las semillas provenientes de Ozumba (humedad 8.02 g/100 g) el componente principal fue la fibra cruda (38.76 g/100 g BS), seguido por carbohidratos (27.62 g/100 g BS), proteínas (21.59 g/100 g BS), lípidos (9.62 g/100 g BS) y ceniza ( $2.41 \mathrm{~g} / 100 \mathrm{~g}$ BS). Mientras que en las semillas provenientes de Tochimilco (humedad $9.99 \mathrm{~g} / 100 \mathrm{~g}$ ), el componente principal fueron los carbohidratos (35.89 g/100 g BS), seguido por la fibra cruda (32.67 g/100 g BS), proteínas (20.42 g/100 g BS), lípidos (8.55 g/100 g BS) y ceniza (2.41 g/100 g BS) (cuadro 2).

En el contenido de cenizas de las semillas de Inga, no hubo diferencia estadísticamente significativa y los valores fueron menores a lo reportado anteriormente para ésta misma semilla (3.05 g/100 g) [26] y para lo encontrado en soya (6.30 g/100 g) [27].

Con respecto al contenido de extracto etéreo, no hubo diferencia estadísticamente significativa en las semillas de Inga provenientes de Ozumba y Tochimilco; sin embargo, fueron considerablemente mayores a lo reportado por Bressani [26], que encontró $0.51 \mathrm{~g} / 100 \mathrm{~g}$ y también mayor al contenido de otras semillas de leguminosas como el frijol $(0.8 \mathrm{~g} / 100 \mathrm{~g})$, la lenteja $(1.3 \mathrm{~g} / 100 \mathrm{~g})$ e inclusive el garbanzo $(6.2 \mathrm{~g} / 100 \mathrm{~g})$ [28], pero inferior a la soya (16.40 g/100 g) [27] especie que por su proporción de grasa se emplea para la extracción de aceite.

Generalmente las leguminosas presentan una baja proporción de lípidos en su composición, por lo que se decidió caracterizar a los ácidos grasos que constituyen a está fracción.

Los valores del contenido de proteína en la semilla de Inga no presentaron diferencia estadísticamente significativa $(p<0.05)$ y está dentro del intervalo que se ha reportado para la mayoría de las semillas de leguminosas, las cuales contienen por lo general alrededor de $22 \%$ de proteína [4], tales como el frijol, garbanzo y lenteja crudos que presentan 22.0, 18.2 y $24.0 \%$ de proteína respectivamente [28].

Cuando se determinó la proporción de carbohidratos en las semillas se encontró que si existían diferencias estadísticamente significativas entre ambas ( $p<0.05)$, lo mismo ocurrió con el contenido de fibra. El contenido de carbohidratos fue menor al reportado para garbanzos $(57.7 \mathrm{~g} / 100 \mathrm{~g})$ y lenteja (57.4 g/100 g) [28]; mientras que el de fibra fue mayor al reportado para estas mismas leguminosas (3 y $0.8 \mathrm{~g} / 100 \mathrm{~g}$ respectivamente) según lo reportado por Asif, Rooney et al. [29].

Dados los antecedentes del uso de las proteínas de origen vegetal, particularmente de las provenientes de leguminosas, así como los resultados obtenidos sobre el contenido proteico de las semillas de Inga, existe la posibilidad de considerarlas como fuente proteica; sin embargo, es necesario conocer la composición de aminoácidos para poder estimar su calidad biológica y biodisponibilidad y por otro lado, es sustancial conocer que compuestos que interfieren en la disponibilidad de las mismas están presentes y la proporción de los mismos para, de ser necesario, considerar un tratamiento que los disminuya o elimine.

\section{Identificación de ácidos grasos}

Se determinó la composición de ácidos grasos presentes en el extracto etéreo de las semillas obtenido por extracción sólido-líquido en soxhlet, mediante cromatografía de gases acoplada a espectrometría de masas (CG-MS). Los resultados obtenidos se muestran en el cuadro 3.

En el aceite de ambas semillas, el ácido palmítico se encuentra en mayor proporción. El palmítico es un ácido graso saturado (AGS) de 16 átomos de Carbono presente en la mayoría de las grasas y aceites. Los AGS más comunes de la dieta proceden principalmente de grasas animales y lácteas; sin embargo, también se han observado niveles considerables en algunos aceites tropicales, especialmente en los aceites de palma y de coco [30], con una proporción similar al aceite de las semillas de Inga.

También está presente el ácido linoleico (LA), ácido graso esencial primario o generador de la familia $\omega-6$ el cual posee18 átomos de carbono y dos dobles enlaces. Se denomina $\omega-6$ debido a que el primer doble enlace se encuentra en el sexto carbono del extremo metilo de la cadena de ácidos grasos. El LA puede ser desaturado y alargado en humanos para formar series de ácidos grasos poliinsaturados (AGPI) $\omega-6$ [31]. El ácido linoleico reduce el colesterol LDL (lipoproteína de baja

\section{CUADRO 2}

Composición química de las harinas de semillas de Inga paterno.

\begin{tabular}{lcc}
\hline & Ozumba & Tochimilco \\
Humedad & $8.02 \pm 0.28^{\mathrm{a}}$ & $9.99 \pm 0.59^{\mathrm{b}}$ \\
Cenizas* $^{*}$ & $2.41 \pm 0.01^{\mathrm{a}}$ & $2.47 \pm 0.04^{\mathrm{a}}$ \\
Extracto etéreo* $^{*}$ & $9.62 \pm 1.45^{\mathrm{a}}$ & $8.55 \pm 0.49^{\mathrm{a}}$ \\
Proteína* $^{\text {Carbohidratos* }}$ & $21.59 \pm 1.40^{\mathrm{a}}$ & $20.42 \pm 0.59^{\mathrm{a}}$ \\
Fibra* $^{*}$ & $27.62 \pm 0.16^{\mathrm{a}}$ & $35.89 \pm 0.05^{\mathrm{b}}$ \\
\hline
\end{tabular}

Los resultados representan la media de tres experimentos independientes \pm desviación estándar * expresado en g/100 g en base seca.

Letras diferentes en la misma fila indican diferencia significativa $(p<0.05)$. 
densidad conocido como "colesterol malo") que es un factor de riesgo para enfermedades cardíacas, también reduce los niveles de triglicéridos en sangre y frena las arritmias cardiacas adversas [32].

La proporción de ácido esteárico fue 9.84 y $9.73 \%$ para los aceites de las semillas de Ozumba y Tochimilco, respectivamente. El esteárico es un ácido graso saturado de cadena larga (18C), presente en la mayoría de grasas y aceites. Es útil como ingrediente en la fabricación de velas, jabones, plásticos y cosméticos.

En el aceite obtenido a partir de las semillas de Ozumba se identificó la presencia de Ácido 7-octadecanoico en una proporción del $8.68 \%$, mientras que para el de las semillas de Tochimilco, la proporción fue de $8.1 \%$. Se trata de un ácido monoinsaturado de 18 átomos de carbono.

El ácido $\alpha$-linolénico (ALA) es el principal precursor de la familia $\omega-3$, los cuales dan lugar a la síntesis de prostaglandinas, tromboxanos, ácidos hidroxiperoxieicosatetraenoico (HPETE) e hidroxieicosatetraenoico (HETE) y leucotrienos, estos compuestos están relacionados con la activación de las plaquetas y leucocitos, estimulan el proceso de coagulación, son constrictores extremadamente potentes de la musculatura lisa, aumentan la permeabilidad vascular y previenen la úlcera gastroduodenal [33], presenta 18 átomos de carbono, pero posee tres dobles enlaces. El LA y el ALA se encuentran en todas las grasas de la dieta y presentan mayores proporciones en la mayoría de los aceites vegetales. El ALA se encuentra sobre todo en las plantas y presenta mayores concentraciones en algunas semillas y frutos secos y en algunos aceites vegetales [31].

La proporción de ácido oleico fue de $1.56 \%$ y $1.66 \%$ para el aceite de Ozumba y de Tochimilco, respectivamente. El oleico es un ácido graso monoinsaturado (AGMI) de la serie $\omega-9$ de 18 átomos de carbono, se ha reportado su presencia en la mayoría de los aceites y grasas, especialmente en el aceite de oliva, de canola, de girasol y de cártamo. Tiene efectos beneficiosos en la regulación del metabolismo de los lípidos y el equilibrio del peso corporal, reduce las lipoproteínas LDL, las cuales actúan de forma negativa sobre las arterias; su principal ventaja al ser consumido es que ayuda de forma inmediata a la salud del corazón y a su adecuado funcionamiento [32].

En el aceite de las semillas de Tochimilco se identificó la presencia de ácido araquídico (0.59\%), ácido graso saturado de 20 átomos de carbono. La fuente más común de este ácido graso es el aceite de cacahuate, aunque puede encontrarse en bajos niveles en los lípidos provenientes de grasas animales [34].

El aceite obtenido a partir de semillas de Inga paterno presentó una proporción semejante de ácido palmítico, un AGS, al del aceite de palma (43\%) [30]. Sin embargo, destaca el contenido de AGNS, como lo son los ácidos 7-octadecanoico, oleico, linoleico y alfa linolénico.

\section{CUADRO 3}

Identificación de los ácidos grasos presentes en el aceite de las semillas de Inga paterno (\%).

\begin{tabular}{lcc}
\hline & Ozumba & Tochimilco \\
Ácido palmítico & $46.57 \pm 0.10^{\mathrm{a}}$ & $37.69 \pm 0.09^{\mathrm{b}}$ \\
Ácido linoleico & $26.34 \pm 0.03^{\mathrm{a}}$ & $31.06 \pm 0.01^{\mathrm{b}}$ \\
Ácido alfa-linolénico & $7.01 \pm 0.06^{\mathrm{a}}$ & $11.17 \pm 0.05^{\mathrm{b}}$ \\
Ácido esteárico & $9.84 \pm 0.09^{\mathrm{a}}$ & $9.73 \pm 0.17^{\mathrm{a}}$ \\
Ácido 7-octadecanoico & $8.68 \pm 0.02^{\mathrm{a}}$ & $8.10 \pm 0.45^{\mathrm{a}}$ \\
Ácido oleico & $1.56 \pm 0.12^{\mathrm{a}}$ & $1.66 \pm 0.14^{\mathrm{a}}$ \\
Ácido araquídico & $\mathrm{ND}$ & $0.59 \pm 0.12$ \\
\hline
\end{tabular}

Los resultados representan la media de tres experimentos independientes.

$N D=$ No Detectado. Letras diferentes en la misma fila indican diferencia significativa $(p<0.05)$.

\section{CUADRO 4}

Compuestos no nutricionales en semillas de Inga paterno.

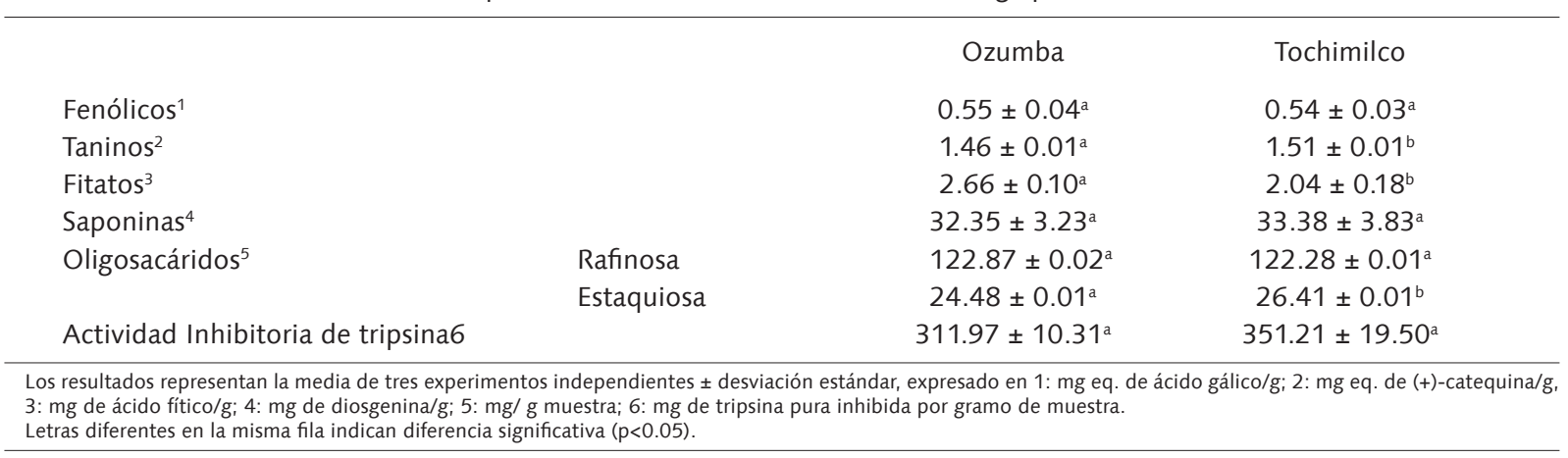




\section{Compuestos no nutricionales}

Se determinó el contenido de compuestos fenólicos, taninos, fitatos, saponinas y la actividad inhibitoria de tripsina de las semillas de las dos regiones (cuadro 4).

El contenido de compuestos fenólicos y de taninos en las semillas de Inga paterno de las dos regiones fue bajo si se compara con lo reportado para otras leguminosas como la lenteja (5.37 $\mathrm{mg} / \mathrm{g}$ respectivamente) [35]. Uno de los inconvenientes de la presencia de estos compuestos al consumirse es la baja disponibilidad de la proteína por formación de complejos e inhibición de algunas enzimas por esta misma razón [5]; sin embargo, también ejercen efectos benéficos a la salud principalmente a través de su actividad antioxidante [36] siendo capaces de eliminar radicales libres, quelar metales y activar enzimas antioxidantes [37].

El contenido de fitatos en las semillas de Inga, fue similar al que se reporta para lenteja $(2.7 \mathrm{mg} / \mathrm{g})$ [35], pero inferior al reportado para el haba $(8.6 \mathrm{mg} / \mathrm{g})$ [38]. La baja disponibilidad de minerales por quelación de los mismos y formación de complejos con proteínas, es uno de los problemas que se relaciona con la presencia de fitatos [39]. Por otro lado, los fitatos juegan un importante papel en el metabolismo de las plantas, y en la resistencia a patógenos, además su presencia en la dieta humana han demostrado acción anticancerígena y una disminución del riesgo de enfermedades del corazón o diabetes $[40,41]$.

Las semillas de Inga presentaron un contenido elevado de saponinas si se compara con lo reportado para lenteja (7.0 $\mathrm{mg} / \mathrm{g}$ ) [42]. Si bien las saponinas eran consideradas como antinutrientes, su consumo ahora es calificado como beneficioso debido al efecto hipocolesterolémico, de prevención de cáncer, de protección en hígado y contra la infección de VIH [43].

Los oligosacáridos que lograron identificarse en las semillas de Inga fueron la rafinosa y la estaquiosa, la verbascosa no fue detectada.

En las semillas de Inga provenientes de Ozumba y Tochimilco se encontró rafinosa en una concentración de $122.87 \pm 0.02$ y $122.28 \pm 0.0 \mathrm{mg} / \mathrm{g}$ respectivamente; en cuanto al contenido de estaquiosa (principal oligosacárido identificado en leguminosas) se obtuvieron valores de $24.48 \pm 0.00$ y $26.41 \pm 0.01 \mathrm{mg} / \mathrm{g}$ muestra, respectivamente. Se han cuantificado oligosacáridos en otras leguminosas como haba encontrándose $3.18 \mathrm{mg}$ rafinosa/g y $0.03 \mathrm{mg}$ estaquiosa/g en los cotiledones [44]. Además, se han reportado contenidos considerables de oligosacáridos en garbanzo, lenteja, lupinus y chícharo en un intervalo de 10 a $120 \mathrm{mg} / \mathrm{g}$ [45].

Si bien, los inconvenientes del consumo de oligosacáridos debido a la falta de $\beta$-galactosidasa se tienen identificados desde hace tiempo (flatulencias, hinchazón, diarrea) [46] y han llegado a limitar el consumo de leguminosas [47], los oligosacáridos también han mostrado ser de valor potencial para la salud inmune [48-50]. El mecanismo de acción aún no está claro; sin embargo, las evidencias apuntan hacia una posible mejora del sistema inmune innato [51]. Aunque actualmente los estudios se centran en azucares presentes en la leche [52, 53], se ha observado que estos metabolitos provenientes de las plantas pueden también desempeñar un papel importante en estas funciones [54, 55].

La actividad de los inhibidores de tripsina presentó valores por sobre de los que se han reportado para lupinus, lenteja, haba, chícharo, garbanzo y Lathyrus spp. (4.25, 54.8, 64.6, $83.8,124$ y $164 \mathrm{mg}$ de tripsina pura inhibida/g de muestra respectivamente), pero por debajo de lo mostrado para el frijol y la soya (576 y $540 \mathrm{mg}$ de tripsina pura inhibida/g de muestra respectivamente) [56]. Los inhibidores de tripsina inducen la hipersecreción de enzimas pancreáticas (tripsina), estimulando la hipertrofia de páncreas, reduciendo la digestión y absorción de proteínas de la dieta. Estas alteraciones negativas en las funciones digestivas dan como resultado menor retención de nitrógeno y azufre y alteraciones en el crecimiento en animales de experimentación [57]. Sin embargo, presentan la interesante característica de tener potencial anticarcinogénico [58]. El nivel de actividad de inhibidor de tripsina en semillas de leguminosas puede reducirse mediante métodos de procesamiento térmico tales como remojo en agua hirviendo, esterilización en autoclave [59], y más recientemente la cocción por extrusión [60].

\section{CONCLUSIÓN}

Las semillas de Inga paterno provenientes de dos regiones de México (Tochimilco, Puebla y Ozumba, Estado de México) no presentaron en general diferencia estadísticamente significativas entre ellas en sus características físicas, químicas y no nutricionales.

Inga paterno es una leguminosa con un contenido de nutrientes similar al que se ha reportado para otras semillas de leguminosa, pudiendo representar una opción de fuente proteica. Las semillas tuvieron un contenido de compuestos no nutricionales menor al (reportado para otras leguminosas en cuanto a fenólicos, taninos y fitatos se refiere, pero la presencia de saponinas e inhibidores de tripsina fue mayor. Los resultados aquí mostrados representan un avance en la caracterización de semillas de leguminosas de bajo consumo y con excelentes opciones de uso alimentario.

\section{RESUMEN}

Inga paterno es una leguminosa arbórea, que crece varios estados de México. La vaina se consume como fruto, debido a la cubierta dulce (Arilo) de la semilla. Ésta última, no es comúnmente consumida. Las características físicas, composición nutricional y el contenido de compuestos no nutricionales presentes en las semillas de Inga paterno provenientes de los Estados de México y Puebla fueron analizados, mostrando que las semillas son de 1.6 y $1.9 \mathrm{~cm}$ largo y 0.9 y $1.08 \mathrm{~cm}$ de ancho. Los componentes químicos principales son: proteína (20.42 y 21.59 g/100 g), carbohidratos (28.99 y $36.15 \mathrm{~g} / 100 \mathrm{~g})$, lípidos (9.62 y $8.55 \mathrm{~g} / 100 \mathrm{~g}$ ) para las procedentes de Ozumba y Tochimilco, respectivamente. Los aceites de las semillas de Inga paterno presentaron una proporción importante de ácido palmítico, así como ácidos 7-octadecanoico, oleico, linoleico y alfa linolénico. Se cuantificaron los compuestos no nutricionales como fenólicos (0.55 y 0.54 $\mathrm{mg}$ eq. de ácido gálico /g), taninos (1.46 y $1.51 \mathrm{mg}$ eq. de (+)-catequina/g), fitatos (2.66 y $2.04 \mathrm{mg}$ de ácido fítico/g), saponinas (32.35 y $33.38 \mathrm{mg}$ de diosgenina/g) e inhibidores de tripsina (311.97 y $351.21 \mathrm{mg}$ de tripsina pura inhibida/g de muestra). Ambas semillas analizadas no presentaron diferencia estadísticamente significativa en sus características físicas, químicas y no nutricionales; sin embargo, éstas presentan una elevada actividad inhibitoria de tripsina comparada con otras leguminosas.

Palabras clave: Leguminosas, inga paterno, composición nutricional, compuestos no nutricionales.

\section{BIBLIOGRAFÍA}

1. FAO, F., Statistical Yearbook 2013: World Food and Agriculture. FAO Food Agric. Organziation UN Rome Italy, 2013.

2. Orhan, I. and B. Sener, Fatty Acid Content of Selected Seed 
Oils. J Herbal Pharmacother. 2002; 2(3): 29-33.

3. Zia-Ul-Haq, M., et al., Nutritional and compositional study of Desi chickpea (Cicer arietinum L.) cultivars grown in Punjab, Pakistan. Food Chem, 2007. 105(4): p. 1357-63.

4. Latham, M.C., Human nutrition in the developing world. Vol. 29. 2002: FAO.

5. Muzquiz, M., Non-nutritional factors in vegetable protein sources: Their involvement in nutrition and health [en línea]. Brazilian J Food Technol [Río de Janeiro, Brasil], 2006 p 87-98.

6. Campos-Vega, R., G. Loarca-Piña, and B.D. Oomah, Minor components of pulses and their potential impact on human health. Food Res Internat, 2010. 43(2): 461-82.

7. Sánchez-Chino, X., et al., Nutrient and Nonnutrient Components of Legumes, and Its Chemopreventive Activity: A Review. Nutr Cancer, 2015. 67(3): 401-10.

8. Martínez, J. and A. Zulet, Legume. Food: composition and properties. Astiasarán I.; J. Martínez (Eds). Editorial McGraw Hill, España, 2003.

9. Navarrete-Tindall, N. and H. Aragon, Inga paterno Harms, in Tropical Tree Seed Manual: Part II, Species Descriptions. Agriculture Handbook2002. p. 523-5.

10. Cordero, J., et al.,Description of species of trees native to Central America. In. Boshier, D. \& Cordero, J. (Eds.) Manual de Arboles de Centroamérica, 2004, Oxford Forestry Institute. Centro Agronómico de Investigación y Enseñanza. Costa Rica. p. 599-613.

11. Ruiz-Carrera, V., et al., Macronutrient phyto-food sources of species exploited by ethnic groups in Tabasco, Mexico. Universidad y Ciencia Número Especial, 2004. 1: p. 27-31.

12. Clegg, K., The application of the anthrone reagent to the estimation of starch in cereals. J Scie. Food Agricult. 1956. 7(1): 40-4.

13. Martin Del Campo Barba, S.T., Identification of authenticity parameters in milk and dairy products, INAPG (AgroParisTech). 2006.

14. Swain, T. and W. Hillis, The phenolic constituents of Prunus domestica. I.-The quantitative analysis of phenolic constituents. J Scie Food Agricult. 1959. 10(1): 63-8.

15. Shahidi, F., M. Naczk, and W. Griffiths, Food Phenolics: Sources, Chemistry, Effects, Applications. Trends Food Sci Technol, 1996. 7(7): 243.

16. Vaintraub, I.A. and N.A. Lapteva, Colorimetric determination of phytate in unpurified extracts of seeds and the products of their processing. Analyt Biochem, 1988. 175(1): 227-30.

17. Smith, C., et al., The determination of trypsin inhibitor levels in foodstuffs. J Sci Food Agriculture, 1980. 31(4): 341-50.

18. Liu, K. and P. Markakis, Trypsin inhibition assay as related to limited hydrolysis of inhibitors. Analyt Biochem, 1989. 178(1): 159-65.

19. Hiai, S., H. Oura, and T. Nakajima, Color reaction of some sapogenins and saponins with vanillin and sulfuric acid. Planta Medica, 1976. 29(2): 116-22.

20. Muzquiz, M., et al., Effect of germination on the oligosaccharide content of lupin species. J Chromatogr A, 1992. 607(2): 349-52.

21. Johansen, H.N., V. Glitsø, and K.E. Bach Knudsen, Influence of Extraction Solvent and Temperature on the Quantitative Determination of Oligosaccharides from Plant Materials by High-Performance Liquid Chromatography. J Agricul Food Chem, 1996. 44(6): 1470-4.

22. Niembro, R.A., Inga jinicuil, in Tropical Tree Seed Manual:
Part II, Species Descriptions. Agriculture Handbook2002. 521-2.

23. Faiguenbaum, M.H. and L. Romero A., Efecto del tamaño de semilla sobre la germinación, el vigor y el rendimiento en un híbrido de maíz (Zea mays L) (Effect of seed size and shape on the germination, vigor and yield of a corn hybrid (Zea maiz). Ciencia Invest Agraria, 1991. 18(3): $111-7$.

24. Shieh, W. and M.B. McDonald, The influence of seed size, shape and treatment on inbred seed corn quality. Seed Sci Technol, 1982. 10: 307-13.

25. NOM-147-SSA1-1996, Cereales y sus productos. Harinas de cereales, semolas o semolinas. Alimentos a base de cereales, de semillas comestibles, harinas, semolas o semolinas o sus mezclas. Productos de panificacion. Disposiciones y especificaciones sanitarias y nutrimentales (Cereals and cereal products. Cereal flour, meal or semolina. Cereal-based foods, edible seeds, flour, semolina or mixtures thereof. Bakery products. Provisions and Sanitary and nutritional specifications). Diario Oficial de la Federación.1999.

26. Bressani, R., Nutritional assessment of chemical seed meal different species of Inga (I. jinicuil, I. Laurina, I. vera). Preliminary studies for incorporation into the diet of the rural population. Universidad del Valle de Guatemala: Guatemala. 2010, 35-8.

27. Menchú, M.T., et al.,Nutritive food value from Centroamerica), INCAP: Guatemala.2000.

28. Foods, L., Food composition table Latin America). Washington, DC: FAO, 2002.

29. Asif, M., et al., Application and opportunities of pulses in food system: a review. Critical Reviews Food sci nutr. 2013. 53(11): 1168-79.

30. FAO, Fats and oils in human nutrition). Food Agricult Org. Vol. 57. 1997.

31. White, P.J., Fatty acids in oilseeds (vegetable oils). Fatty acids in foods and their health implications, 2007: p. 253-5.

32. Oh, K., et al., Dietary Fat Intake and Risk of Coronary Heart Disease in Women: 20 Years of Follow-up of the Nurses' Health Study. Am J Epidemiol, 2005. 161(7): 672-9.

33. Teitelbaum, J.E. and W. Allan Walker, Review: the role of omega 3 fatty acids in intestinal inflammation. J Nutr. Biochem. 2001; 12(1): 21-32.

34. Gebhardt, S., et al., USDA national nutrient database for standard reference, release 21. 2008.

35. Xu, B., S. Yuan, and S. Chang, Comparative analyses of phenolic composition, antioxidant capacity, and color of cool season legumes and other selected food legumes. J Food Sci. 2007; 72(2): S167-S177.

36. Fang, Y.-Z., S. Yang, and G. Wu, Free radicals, antioxidants, and nutrition. Nutrition, 2002. 18(10): 872-9.

37. Heim, K.E., A.R. Tagliaferro, and D.J. Bobilya, Flavonoid antioxidants: chemistry, metabolism and structure-activity relationships. J Nutr Biochem, 2002. 13(10): 572-84.

38. Burbano, C., et al., Determination of phytate and lower inositol phosphates in Spanish legumes by HPLC methodology. Food Chem, 1995. 52(3): 321-5.

39. Martínez-Domínguez, B., M.V. Ibáñez-Gómez, and F. Rincón-León, Phytic acid: nutritional aspects and analytical implications. Arch Latinoam Nutr. 2002; 52: 219-31.

40. Vucenik, I. and A.M. Shamsuddin, Protection against cancer by dietary IP6 and inositol. Nutr Cancer, 2006. 55(2): 109-25.

41. Marie Minihane, A. and G. Rimbach, Iron absorption and the iron binding and anti-oxidant properties of phytic acid. International J Food Science Technol, 2002. 37(7): 741-8. 
42. Srivastava, $R$. and $H$. Vasishtha, Saponins and lectins of Indian chickpeas (Cicer arietinum) and lentils (Lens Culinaris). Indian J Agricul Biochem, 2012. 25(1): p. 44-7.

43. Shi, J., et al., Saponins from edible legumes: chemistry, processing, and health benefits. J Medi Food, 2004. 7(1): 67-78.

44. Valdez-Anguiano, D.M., et al., Changes of Phenolic Compounds and Antioxidant Activity Induced by Germination of Vicia faba, in Seeds as Functional Foods and Nutraceuticals2014. p. 143-56.

45. Saini, H.S., Legume seed oligosaccharides, in Recent Advances of Research in Antinutritional Factors in Legume Seeds, J. Huisman, Van der Poel, T. F. B., Liener, I. E., Editor 1989. p. 329-341.

46. Cristofaro, E., F. Mottu, and J. Wuhrmann, Involvement of the raffinose family of oligosaccharides in flatulence. Sugars in Nutrition. HL Sipple \& KW McNutt, eds, 1974.

47. Schneider, A.V., Overview of the market and consumption of puises in Europe. British J Nutr. 2002. 88(S3): 243-50.

48. Eiwegger, T., et al., Human milk-derived oligosaccharides and plant-derived oligosaccharides stimulate cytokine production of cord blood T-cells in vitro. Pediatric Res, 2004. 56(4): 536-40.

49. Swanson, K., et al., Effects of Supplemental Fructooligosaccharides Plus Mannanoligosaccharides on Immune Function and Ileal and Fecal Microbial Populations in Adult Dogs. Archiv für Tierernaehrung, 2002. 56(4): 309-18.

50. Buddington, R., et al., Non-digestible oligosaccharides and defense functions: lessons learned from animal models. British J Nutr. 2002. 87(S2): S231-9.
51. Lindsay, J.O., et al., Clinical, microbiological, and immunological effects of fructo-oligosaccharide in patients with Crohn's disease. Gut, 2006. 55(3): p. 348-355.

52. Bruzzese, E., et al., Impact of prebiotics on human health. Digestive Liver Dis, 2006. 38: S283-S87.

53. Oddy, W.H., The impact of breastmilk on infant and child health. Breastfeeding Rev, 2002. 10(3): 5.

54. Kaur, N. and A.K. Gupta, Applications of inulin and oligofructose in health and nutrition. J Biosci, 2002. 27(7): 703-14.

55. Stahl, B., L. M'Rabet, and A. Vos, Immunomodulating oligosaccharides, and therapeutic use there of. Int. Patent WO, 2005. 2: 005-039.

56. Guillamón, E., et al., The trypsin inhibitors present in seed of different grain legume species and cultivar. Food Chem. 2008.; 107(1): 68-74.

57. Norton, G., Proteinase inhibitors, in Toxic Substances in Crop Plants, J.P.F. D'Mello, Duffus, C.M., Duffus, J.H., Editor 1991, Toxic Substances in Crop Plants. The Royal Society of Chemistry: Cambridge. p. 68-106.

58. Kennedy, A.R., The Bowman-Birk inhibitor from soybeans as an anticarcinogenic agent. The Am J Clini Nutr. 1998. 68(6): 1406S-2S.

59. Vidal-Valverde, C., et al., Influence of Processing on Trypsin Inhibitor Activity of Faba Beans and Its Physiological Effect. J Agricul Food Chem, 1997. 45(9): 3559-64.

60. Alonso, R., A. Aguirre, and F. Marzo, Effects of extrusion and traditional processing methods on antinutrients and in vitro digestibility of protein and starch in faba and kidney beans. Food Chem, 2000. 68(2): 159-65. 